\title{
INVESTIGACIONES
}

\section{Movilidad escolar en Chile. Análisis de las implicancias para la calidad y equidad de la educación*}

\author{
School mobility in Chile. Analysis of the implications for \\ the quality and equity of education \\ Mobilidade escolar no Chile. Análise das implicações para a
qualidade ea eqüidade da educação
}

\author{
Guillermo Zamora \\ Académico del Departamento de Teoría y Política de la Educación, \\ Facultad de Educación, Pontificia Universidad Católica de Chile. Profesor de Filosofía. \\ Doctor en Ciencias de la Educación. E-mail: gzamora@puc.cl
}

\begin{abstract}
RESUMEN
La movilidad escolar es un fenómeno altamente recurrente en Chile. Anualmente el porcentaje de estudiantes que se trasladan de una escuela a otra llega al 11,5\% en enseñanza básica. Considerando la alta recurrencia, cabe entonces preguntarse: ¿qué implicancias tiene la movilidad escolar en la calidad y equidad de la educación chilena? Con este fin, el estudio realiza un diagnóstico de los aprendizajes que logran los estudiantes de $4^{\circ}$ año de enseñanza básica que se han trasladado de un establecimiento a otro, comparándolos con aquellos estudiantes que permanecen en una misma escuela. También se examina la calidad de los establecimientos que reciben a los que se trasladan. Se trabaja con datos censales de 182.885 estudiantes.
\end{abstract}

Palabras clave: movilidad escolar, calidad educativa, equidad educativa.

\begin{abstract}
The school mobility is a highly recurrent phenomenon in Chile. Every year the percentage of students who move from one school to another reaches $11.5 \%$ in Primary Education. Considering the high recurrence, the question is: What are the implications of school mobility on the quality and equity of the Chilean education? To answer this question, this research issues a diagnosis of the learning achieved by students in 4th grade of Primary Education who have moved from one school to another, comparing them with the learning achievements from those who remained in the same school. It also evaluates the quality of the educational establishments that receives the transferred students. It works with census data from 182885 students.
\end{abstract}

Key words: school mobility, quality of education, equity of education.

\section{RESUMO}

A mobilidade escolar é um fenômeno muito recorrente no Chile. Todos os anos, a percentagem de alunos que mudam de uma escola à outra atinge $11,5 \%$ na Educação Básica. Considerando a alta recorrência, pode-se então perguntar: quais são as implicações da mobilidade escolar sobre a qualidade e a eqüidade da educação chilena? Para este fim, o estudo faz um diagnóstico da aprendizagem alcançada de alunos de $4^{\circ}$ ano do Ensino Básico que se mudaram de um estabelecimento para outro, comparando-a com a aprendizagem alcançada de alunos que permaneceram na mesma escola. Também examina A qualidade dos estabelecimentos que acolhem esses alunos também é examinada Trabalha-se com dados de censo de 182885 estudantes.

Palavras-chave: mobilidade escolar, qualidade educativa, eqüidade educativa.

* $\quad$ Este estudio forma parte de la investigación "Barreras para el aprendizaje y la participación asociadas a la desvinculación escolar. Un estudio acerca de la inclusión educativa en Chile", financiada por CONICYT a través del proyecto FONDECYT No 11090342. 


\section{INTRODUCCION Y ANTECEDENTES}

\section{1. ¿QUE ES LA MOVILIDAD ESCOLAR Y CUAL ES SU MAGNITUD EN CHILE?}

La movilidad escolar se entiende como el retiro del estudiante de su liceo o colegio y su traslado a otro. Ella puede ser forzada por el establecimiento, como también voluntaria. La desvinculación forzada suele presentarse como expulsión o cancelación de matrícula debido a razones económicas, académicas, problemas conductuales, etc. El retiro voluntario corresponde a una decisión autónoma de la familia y puede responder a múltiples situaciones: insatisfacción con el establecimiento, cambio de domicilio, etc.

La movilidad escolar es un fenómeno recurrente en Chile. Los estudios de Sanclemente (2008) y Perticara \& Sanclemente (2009), a partir de la Base de Datos del Registro de Estudiantes de Chile (RECH, 2003 al 2007), constatan que anualmente la proporción de alumnos trasladados o tasa de movilidad llega al 11,5\% en enseñanza básica (primaria). Esta cifra dobla a la de países desarrollados: en Bélgica e Inglaterra la movilidad escolar alcanza el $6 \%$ en este nivel. El estudio de Sanclemente (2008) identifica que sexto básico es el grado donde la movilidad escolar es más alta (15\%). En cuanto a los grupos socioeconómicos (GSE), la mayor movilidad se presenta en los establecimientos que atienden a los GSE medio alto, medio y medio bajo (10,8\%; $10,1 \%$ y $10,2 \%$ en E.B). Menor movilidad se presenta en los grupos extremos, lo cual en el caso del GSE bajo es interpretado por Perticara \& Sanclemente (2009) como una suerte de "trampa de la pobreza”, donde el que llega ahí le es difícil trasladarse.

\section{2. ¿CUALES SON LOS EFECTOS DE LA MOVILIDAD ESCOLAR? ¿POR QUE ES RELEVANTE SU ESTUDIO?}

En cuanto a los efectos de la movilidad escolar hay diversas perspectivas. Eyzaguirre y Fontaine (2008) señalan que la posibilidad de desplazarse de un establecimiento a otro es positiva para la calidad de la educación en cuanto se puede hacer una trayectoria desde un colegio de peor calidad a otro mejor. Ello conduce al mejor aprendizaje de los alumnos y a una mayor competencia entre las escuelas por atraer y retener estudiantes, lo cual mejora la eficiencia de los establecimientos. Hay que considerar que en Chile los padres pueden elegir libremente el establecimiento de sus hijos y el Estado subsidia la demanda a aquellos que asisten a establecimientos municipalizados o particulares subvencionados. Este sistema de financiamiento está vigente desde inicios de la década del 80. Fue implementado por el Gobierno Militar, dentro de una política de liberalización y descentralización del sistema educacional. Consiste en que la subvención estatal sigue al alumno y se transfiere al establecimiento que él y su familia elijan. Si el estudiante se retira, el establecimiento pierde la subvención. Este sistema opera sobre diversos supuestos. Por una parte, se supone que las familias son consumidores racionales de la educación y que deciden su permanencia o retiro bajo evaluaciones de costo y beneficio (Friedman \& Friedman, 1980; Aedo \& Sapelli, 2001). En palabras de Fontaine \& Eyzaguirre (2001): "El modelo asume que los padres buscarán maximizar el nivel de aprendizaje de sus hijos y para identificar las mejores alternativas adquirirán un conocimiento certero de los costos y de los logros que podría tener su hijo en cada establecimiento" (p. 70). Un segundo supuesto es que la demanda de los padres empujará a que se desarrolle un 
mercado educacional donde los mejores establecimientos lograrán atraer a la mayor parte de la demanda y los peores tenderán a desaparecer por no ser elegidos. En palabras de Friedman (1980): "Si el consumidor tiene libertad para elegir, una empresa puede crecer solamente si produce algo que éste prefiere debido a su calidad o precio" (p. 219).

En contraposición a esta perspectiva, cabe considerar otros planteamientos que dan cuenta que la alta movilidad escolar puede ser un síntoma de graves falencias de equidad en el sistema escolar. Se puede plantear que la movilidad escolar es también producto de la alta selección que opera en el sistema educativo, especialmente de los estudiantes menos aventajados. Hay que recordar que en el sistema escolar chileno los establecimientos seleccionan al inicio y durante la trayectoria escolar producto de la fuerte competencia por mostrar mejores indicadores o por mejorar su prestigio social. De este modo, la alta movilidad puede ser expresión de una discriminación escolar, con sus correspondientes dificultades para el aprendizaje y la inclusión de todos los estudiantes, especialmente de los más vulnerables. Al respecto, el informe de la OCDE (2004) señala que las escuelas chilenas, especialmente las particulares subvencionadas, realizan diversos procesos de selección al ingreso y durante la permanencia, expulsando a los alumnos que presentan bajo rendimiento o problemas de conducta.

Hay que sumar que a las dificultades para el aprendizaje se agregan también problemas para la cohesión social. Peña (2007) indica que las selecciones que invocan razones académicas encubren una selección social pues las aptitudes que son calificadas como condiciones mínimas de permanencia dependen de la herencia social y cultural. En esta línea, García Huidobro (2007) señala que las selecciones generadas desde la escuela minan una educación democrática e inclusiva, impiden la mixtura social, restringen la libertad de los padres y no garantizan a todos por igual el derecho a la educación. En cuanto a los efectos para el aprendizaje, Manzi (2007) señala que la evidencia internacional muestra que una escuela inclusiva, donde conviven alumnos de diversos logros de aprendizajes, en oposición a la que realiza selecciones, tiene diversos efectos positivos: beneficia en sus aprendizajes a los grupos de menor rendimiento, favorece la interdependencia entre estudiantes de distinto nivel de logro y brinda experiencias de integración que reducen el riesgo de actitudes negativas hacia grupos desaventajados.

Teniendo presente los efectos negativos de la selección escolar es posible comprender la relevancia de las políticas educativas que buscan favorecer la permanencia de los estudiantes en sus establecimientos. Por ejemplo, la Ley General de la Educación, promulgada el 2009, pretende reducir las discriminaciones arbitrarias prohibiendo la cancelación de la matrícula, suspensión o expulsión debido a embarazo o maternidad, no pago de obligaciones económicas o por el rendimiento de los alumnos (artículo $\mathrm{N}^{\mathrm{o}}$ 11). A su vez, la ley de subvención escolar preferencial, promulgada a inicios de 2008, condiciona esta subvención a aquellos establecimientos que retengan a sus estudiantes desde primer nivel de transición y sexto básico, sin cancelarle la matrícula por rendimiento escolar.

Como se aprecia, la movilidad escolar constituye una problemática que tiene un profundo impacto en los aprendizajes de todos los estudiantes y en la equidad de la educación chilena. Algunos argumentos apuestan a que la movilidad escolar es beneficiosa para la calidad general del sistema, en cuanto los estudiantes pueden trasladarse a establecimientos de mejor calidad, desapareciendo así los peores. Bajo esta perspectiva, una alta tasa de movilidad podría ser síntoma de un saludable sistema educativo. Sin 
embargo, desde otra perspectiva, la alta tasa de movilidad puede encubrir una importante proporción de desvinculaciones forzadas, con las consiguientes dificultades en el aprendizaje y la cohesión social, en cuanto cada establecimiento tiende a atender a una población social cada vez más homogénea.

Considerando estos planteamientos, que algunas veces tienen más componentes ideológicos que empíricos, es que actualmente se hace necesario aportar evidencias científicas al debate. El presente estudio se orienta justamente en esta línea: examinar las implicancias de la movilidad escolar en la calidad y equidad de la educación chilena. Para ello se realiza una caracterización social y educativa de los estudiantes que se movilizan en el primer ciclo básico (hasta $4^{\circ}$ de enseñanza básica) y de la calidad de los establecimientos que los reciben.

\subsection{INTERROGANTES DEL ESTUDIO}

La interrogante general que mueve al presente estudio es:

¿Qué implicancias tiene la movilidad escolar en la calidad y equidad de la educación?

Particularmente, interesa responder las siguientes preguntas:

¿Cuántos y quiénes son los estudiantes del primer ciclo básico que se trasladan de establecimiento? ¿Qué nivel de aprendizaje logran los estudiantes que se trasladan y cuánto difieren con respecto a los que permanecen? ¿Qué establecimientos reciben a los estudiantes que se trasladan? ¿Qué calidad educativa ofrecen?

\subsection{OBJETIVOS}

a) Identificar las características socioeducativas de los estudiantes que se trasladan diferenciándolos de aquellos que permanecen en un mismo establecimiento.

b) Examinar los niveles de aprendizajes que logran los estudiantes que se trasladan y comparar sus diferencias con los que permanecen.

c) Examinar la calidad educativa de los establecimientos que reciben a los estudiantes que se trasladan.

\section{METODOLOGIA}

\subsection{DISEÑO}

El diseño del estudio es de tipo descriptivo y correlacional.

El estudio se propone describir las características socioeducativas de los estudiantes que se trasladan y de los establecimientos que los reciben. Asimismo, se propone examinar las relaciones entre cantidad de estudiantes trasladados y calidad educativa que ofrecen los establecimientos.

Es importante enfatizar que este estudio sólo tiene el propósito de establecer descripciones y relaciones entre variables. El estudio no pretende sugerir una relación lineal, de causa y efecto, entre movilidad escolar y desempeño académico. 


\subsection{SUJETOS DE ESTUDIO Y PROCEDIMIENTO DE OBTENCION DE LA INFORMACION}

La información de este estudio ha sido obtenida de la Base de Datos del Sistema de Medición de Calidad de la Educación (SIMCE 2007) de $4^{\circ}$ E.B. Se consideran las respuestas del total de estudiantes que rinden la prueba y que tienen información para las diversas variables del presente estudio. Cabe señalar que la información examinada proviene tanto de las pruebas SIMCE desarrolladas por los estudiantes, como de los cuestionarios a los padres y apoderados. La cantidad total de estudiantes examinados es de 182.885 .

Las pruebas SIMCE se tomaron los días 13 y 14 de noviembre del 2007. El cuestionario de los padres y/o apoderados se responde en los hogares, en forma anónima y voluntaria, y es entregado en sobre cerrado al establecimiento y supervisor del SIMCE. Su aplicación corresponde a los mismos días en que los alumnos responden las pruebas.

\subsection{OPERACIONALIZACION DE LAS VARIABLES}

a) Variable principal: Movilidad escolar

Se consideran las respuestas de los apoderados al cuestionario SIMCE (pregunta $\mathrm{N}^{\mathrm{o}} 14$, curso en que ingresó su hijo-a-). A partir de ésta se establecen dos categorías:

Estudiantes de $4^{\circ}$ E.B. que permanecen en el mismo establecimiento. Son los alumnos(as) que comienzan su educación obligatoria en el mismo establecimiento donde rinden el SIMCE de $4^{\circ}$ E.B. Es decir, permanecen en el mismo colegio desde $1^{\circ}$ E.B. o antes.

Estudiantes de $4^{\circ}$ E.B. que se han trasladado de establecimiento. Son los alumnos(as) que comienzan su educación obligatoria en un establecimiento distinto de donde rinden el SIMCE. Es decir, se han movilizado de colegio en $2^{\circ}, 3^{\circ}$ o $4^{\circ}$ E.B.

b) Variables de caracterización socioeducativa del estudiante

- Género.

- Años de escolaridad del padre/padrastro y la madre/madrastra.

- Ingreso del hogar

- Desempeño individual del estudiante en las prueba de Lenguaje y Comunicación.

Cabe destacar que se ha privilegiado el desempeño del SIMCE en Lenguaje y Comunicación puesto que es un dominio básico y fundacional en el proceso educativo del estudiante del primer ciclo básico. También es importante destacar que este dominio posibilita el acceso a otras múltiples dimensiones del proceso educativo. Al respecto, en esta prueba la correlación entre Lenguaje y Comunicación y Matemática alcanza a un $\mathrm{r}$ de 0.781 (sig. 0.000) y con Comprensión del Medio Natural a un r de 0.787 (sig. 0.000).

c) Variables de caracterización socioeducativa del establecimiento

- Grupo socioeconómico (GSE). Los GSE son los siguientes, según categoría proporcionada por SIMCE. 


\begin{tabular}{|l|l|l|l|c|}
\hline \multirow{2}{*}{$\begin{array}{c}\text { Grupo } \\
\text { Socieoconómico }\end{array}$} & \multicolumn{2}{|c|}{ Años de escolaridad } & \multicolumn{1}{c|}{$\begin{array}{c}\text { Ingreso del } \\
\text { hogar en U\$ }\end{array}$} & $\begin{array}{c}\text { Índice de vulnerabilidad } \\
\text { escolar (IVE) }\end{array}$ \\
\cline { 2 - 3 } Bajo & Menos de 9 & Menos de 9 & 0 a 257 & $60,01 \%$ y más \\
\hline Medio Bajo & $9-10$ & $9-10$ & 258 a 413 & $37,51 \%-60 \%$ \\
\hline Medio & $11-12$ & $11-12$ & 414 a 721 & $20,01 \%-37,5 \%$ \\
\hline Medio Alto & $13-14$ & $13-15$ & 722 a 1.538 & $0,01 \%-20 \%$ \\
\hline Alto & Más de 14 & Más de 15 & 1.539 y más & $0 \%$ \\
\hline
\end{tabular}

- Dependencia del establecimiento: Municipalizado, Particular Subvencionado y Particular Pagado.

- Promedio SIMCE Lenguaje y Comunicación del establecimiento.

Un primer análisis considera el promedio SIMCE de todos los estudiantes (los que se trasladan y los que permanecen) y la ubicación del establecimiento en comparación con otros del mismo GSE. Se categorizan tres grupos: los establecimientos que tienen promedio significativamente inferior a sus pares; los que no tienen diferencia y los que tienen una diferencia significativamente superior.

Un segundo análisis considera el desempeño SIMCE sólo de los alumnos que han permanecido en el mismo establecimiento desde $1^{\circ}$ E.B. o antes. Se establecen para cada GSE tres niveles de desempeño: Bajo, establecimientos que llegan hasta el percentil 33; Mediano, entre percentil 34 y 66; Alto, superior al percentil 66. Estas categorías han sido elaboradas por el investigador.

\subsection{TECNICA DE ANALISIS}

Se realizan análisis de frecuencias y de correlaciones. También se desarrollan pruebas de diferencias de grupos utilizando chi-cuadrado $\left(\chi^{2}\right)$ y t de Student.

\section{RESULTADOS}

\subsection{CARACTERISTICAS DE LOS ESTUDIANTES}

Los estudiantes que cursan $4^{\circ}$ E.B han ingresado a sus actuales establecimientos (ver tabla 1) predominantemente en los niveles de educación parvularia (especialmente en kinder, a los 5 años). El $68 \%$ de los estudiantes de $4^{\circ}$ E.B. ha permanecido en el mismo establecimiento desde, o incluso antes, del inicio de la escolaridad obligatoria ( $1^{\circ}$ E.B.). A pesar de que la mayoría permanece, es igualmente relevante la cantidad de niños y niñas que se han trasladado de un colegio a otro en el primer ciclo básico: 50.348 estudiantes, que representa el $27,5 \%$ de la población escolar de este nivel. Hay que recordar que este análisis considera sólo el primer ciclo básico donde las experiencias escolares tienen mayor impacto en las trayectorias educativas. 
También es relevante constatar que el porcentaje de trasladados aumenta en la medida que se avanza en los niveles escolares obligatorios: se han trasladado en $2^{\circ}$ E.B. el 7,6\%; en $3^{\circ}$ E.B. el $8,6 \%$ y en $4^{\circ}$ E.B. el $11,3 \%$. Esta tendencia es consistente a lo constatado por Sanclemente (2008) y Perticara \& Sanclemente (2009) que indican que la tasa promedio de movilidad anual en enseñanza básica es de un 11,5\%. Reunidos este dato y los anteriores, se podría estimar, en forma hipotética, que al término de la enseñanza básica ( $8^{\circ}$ E.B) la mayoría de los estudiantes experimentarán el traslado de un establecimiento a otro, siendo los menos los que permanezcan en el mismo colegio en donde comenzaron su educación obligatoria.

\section{Tabla 1}

Curso de ingreso al establecimiento de los estudiantes que cursan $4^{\circ}$ E.B.

\begin{tabular}{|c|c|c|c|c|}
\hline & \multirow{2}{*}{$\begin{array}{c}\text { Frecuencia } \\
24.245\end{array}$} & \multirow{2}{*}{$\begin{array}{c}\text { Porcentaje } \\
13,3 \\
\end{array}$} & \\
\hline \multirow[t]{9}{*}{ Válido } & Prekinder & & & \\
\hline & Kinder & 66.492 & $36,4\}$ & \multirow[t]{3}{*}{$68,0 \%$ permanecen } \\
\hline & $1^{\circ} \mathrm{EB}$ & 33.596 & 18,4 & \\
\hline & $2^{\circ} \mathrm{EB}$ & 13.969 & 7,6 & \\
\hline & $3^{\circ} \mathrm{EB}$ & 13.969 & $8,6\}$ & \multirow[t]{5}{*}{$27,5 \%$ se trasladan } \\
\hline & $4^{\circ} \mathrm{EB}$ & 20.636 & 11,3 & \\
\hline & Vacío & 4.159 & 2,3 & \\
\hline & Doble marca & 4.045 & 2,2 & \\
\hline & Total & 182.885 & 100,0 & \\
\hline
\end{tabular}

En cuanto a las características socioeducativas (ver tabla 2), los estudiantes que se trasladan son en una mayor proporción hombres y sus padres y madres tienen más años de escolaridad que los estudiantes que permanecen. Cabe destacar que las diferencias al respecto son estadísticamente significativas, aunque no sustantivas. En cuanto al ingreso mensual, no hay diferencias estadísticamente significativas entre ellos.

Con respecto al desempeño de los estudiantes (ver tabla 3), se puede constatar que aquellos que se trasladan tienden a presentar, en forma significativa, menores logros en sus aprendizajes en la prueba SIMCE que los que permanecen: 252,00 y 257.74 puntos respectivamente, lo que representa una diferencia de 5,74 puntos. Las diferencias son estadísticamente significativas y se presentan en las tres dependencias y en los cinco GSE.

La mayor distancia entre el puntaje de los que se trasladan y los que permanecen se presenta en los establecimientos particulares pagados (9,26 puntos). Más aún, en el GSE medio alto de esta dependencia se presenta la mayor diferencia de todos los sectores $(15,28$ puntos). Y al contrario, la dependencia que presenta menor diferencia es la municipalizada (5,88 puntos). Aquí, incluso, el GSE medio alto no presenta diferencia estadísticamente significativa. 


\section{Tabla 2}

\section{Características socioeducativas}

\begin{tabular}{|l|l|l|l|c|}
\cline { 2 - 5 } \multicolumn{1}{c|}{} & \multicolumn{1}{c|}{$\begin{array}{c}\text { Estudiantes que } \\
\text { permanecen }\end{array}$} & $\begin{array}{c}\text { Estudiantes que } \\
\text { se trasladan }\end{array}$ & $\begin{array}{c}\text { Total } \\
\text { población }\end{array}$ & $\begin{array}{c}\text { Prueba de diferencia } \\
\text { (permanecen/se movilizan) }\end{array}$ \\
\hline Género & $\begin{array}{l}\text { Mujeres: } 50,1 \% \\
\text { Hombres: } 49,9 \%\end{array}$ & $\begin{array}{l}\text { Mujeres: } 48,8 \% \\
\text { Hombres: } 51,2 \%\end{array}$ & $\begin{array}{l}\text { Mujeres: } 49,7 \% \\
\text { Hombres: } 50,3 \%\end{array}$ & $\begin{array}{l}\chi^{2}=23,949 \\
\text { Sig. }=0,000\end{array}$ \\
\hline $\begin{array}{l}\text { Años promedio escolaridad } \\
\text { del padre/padrastro }\end{array}$ & $\begin{array}{l}10,16 \text { años } \\
(\mathrm{DS}=2,61)\end{array}$ & $\begin{array}{l}10,40 \text { años } \\
(\mathrm{DS}=2,51)\end{array}$ & $\begin{array}{l}10,20 \text { años } \\
\text { (DS 2,61) }\end{array}$ & $\begin{array}{l}\mathrm{t}=-18,94 \\
\text { Sig. }=0,000\end{array}$ \\
\hline $\begin{array}{l}\text { Años promedio escolaridad } \\
\text { de la madre/madrastra }\end{array}$ & $\begin{array}{l}10,17 \text { años } \\
(\mathrm{DS}=2,59)\end{array}$ & $\begin{array}{l}10,33 \text { años } \\
(\mathrm{DS}=2,53)\end{array}$ & $\begin{array}{l}10,18 \text { años } \\
\text { (DS 2,59) }\end{array}$ & $\begin{array}{l}\mathrm{t}=-12,76 \\
\text { Sig. }=0,000\end{array}$ \\
\hline $\begin{array}{l}\text { Ingreso promedio mensual } \\
\text { (familia) }\end{array}$ & $\begin{array}{l}\text { Entre U\$600 y } \\
\mathrm{U} \$ 800\end{array}$ & $\begin{array}{l}\text { Entre U\$ 600 y } \\
\mathrm{U} \$ 800\end{array}$ & $\begin{array}{l}\text { Entre U\$ 600 y } \\
\text { U\$ 800 }\end{array}$ & \\
\hline
\end{tabular}

\section{Tabla 3}

Puntaje SIMCE considerando dependencia y GSE

\begin{tabular}{|c|c|c|c|c|c|c|c|}
\hline \multicolumn{2}{|c|}{ Dependencia/GSE } & Bajo & Medio bajo & Medio & Medio alto & Alto & Total \\
\hline Municipalizado & $\begin{array}{l}\text { Permanecen } \\
\text { Se trasladan } \\
\text { Diferencia }\end{array}$ & $\begin{array}{l}243.27 \\
234.63 \\
\\
8.64 \\
t=7.51 \\
\text { Sig. }=0,000\end{array}$ & $\begin{array}{l}240.18 \\
231.99 \\
\\
8.19 \\
\mathrm{t}=14.62 \\
\text { Sig. }=0,000\end{array}$ & $\begin{array}{l}250.69 \\
247.12 \\
3.27 \\
t=4.98 \\
\text { Sig. }=0,000\end{array}$ & $\begin{array}{l}277.14 \\
277.84 \\
-0.7 \\
t=-0.337 \\
\text { Sig. }=0,736\end{array}$ & & $\begin{array}{l}244.87 \\
328.99 \\
5.88 \\
t=14,41 \\
\text { Sig. }=0,000\end{array}$ \\
\hline P. subvencionado & $\begin{array}{l}\text { Permanecen } \\
\text { Se trasladan } \\
\text { Diferencia }\end{array}$ & $\begin{array}{l}233.90 \\
227.16 \\
\\
6.74 \\
t=4.03 \\
\text { Sig. }=0,000\end{array}$ & $\begin{array}{l}243.86 \\
236.68 \\
\\
7.18 \\
t=7.07 \\
\text { Sig. }=0,000\end{array}$ & $\begin{array}{l}261.38 \\
253.86 \\
\\
7.52 \\
\mathrm{t}=13.10 \\
\text { Sig. }=0,000\end{array}$ & $\begin{array}{l}281.15 \\
275.70 \\
5.45 \\
t=8.66 \\
\text { Sig. }=0,000\end{array}$ & $\begin{array}{l}294.35 \\
289.21 \\
\\
2.14 \\
\mathrm{t}=2.24 \\
\text { Sig. }=0,029\end{array}$ & $\begin{array}{l}265.67 \\
258.28 \\
7.39 \\
t=18,87 \\
\text { Sig. }=0,000\end{array}$ \\
\hline P. pagado & $\begin{array}{l}\text { Permanecen } \\
\text { Se trasladan } \\
\text { Diferencia }\end{array}$ & & & & $\begin{array}{l}279.63 \\
264.35 \\
15.28 \\
t=2.93 \\
\text { Sig. }=0,004\end{array}$ & $\begin{array}{l}302.26 \\
294.81 \\
7.45 \\
t=6.97 \\
\text { Sig. }=0,000\end{array}$ & $\begin{array}{l}301.67 \\
292.41 \\
9.26 \\
t=9,23 \\
\text { Sig. }=0,000\end{array}$ \\
\hline Total & $\begin{array}{l}\text { Permanecen } \\
\text { Se trasladan } \\
\text { Diferencia }\end{array}$ & $\begin{array}{l}241.66 \\
231.87 \\
9.79 \\
t=10.54 \\
\text { Sig. }=0,000\end{array}$ & $\begin{array}{l}240.83 \\
233.21 \\
\\
7.62 \\
t=15.60 \\
\text { Sig. }=0,000\end{array}$ & $\begin{array}{l}256.88 \\
251.31 \\
\\
5.57 \\
t=12.31 \\
\text { Sig. }=0,000\end{array}$ & $\begin{array}{l}280.78 \\
275.62 \\
\\
5.16 \\
t=8.59 \\
\text { Sig. }=0,000\end{array}$ & $\begin{array}{l}301.21 \\
293.70 \\
7.51 \\
t=7.73 \\
\text { Sig. }=0,000\end{array}$ & $\begin{array}{l}257,74 \\
252,00 \\
\\
5.74 \\
t=20,28 \\
\text { Sig. }=0,000\end{array}$ \\
\hline
\end{tabular}




\subsection{CARACTERISTICAS DE LOS ESTABLECIMIENTOS DONDE ASISTEN LOS ESTUDIANTES QUE SE TRASLADAN}

Los colegios particulares subvencionados son los que reciben el mayor porcentaje de estudiantes que se trasladan, 52,4\%; los municipalizados reciben al 42,2\% y los particulares pagados al 5,4\% (ver tabla 4). Estos porcentajes son relativamente consistentes con la distribución general de la población en las diversas dependencias: el 45,9\% de los estudiantes de cuarto básico asisten a establecimientos particulares subvencionados; el $47,7 \%$ a municipalizados y el $6,4 \%$ a particulares pagados.

También es importante señalar que los establecimientos del GSE medio son los que reciben la mayor cantidad de estudiantes trasladados: el 86,7\% de todos los que se trasladan asisten a establecimientos del GSE medio bajo, medio y medio alto. Estos porcentajes también son consistentes con la distribución general de la población: el 9,2\% de los estudiantes está matriculado en establecimientos clasificados como GSE Bajo, el 31,2\% en Medio Bajo, el 35,3\% en Medio, el 17,2\% en Medio Alto y el 7,1\% en Alto.

Para controlar el efecto del tamaño de la población en la forma en que se distribuyen los trasladados, a continuación se examina la proporción de estudiantes trasladados que atiende cada dependencia y GSE (ver tabla 5). Con este análisis se logrará identificar los establecimientos que reciben, proporcionalmente, más estudiantes trasladados.

De acuerdo a los datos de la tabla 5, el sector particular subvencionado atiende, proporcionalmente, más estudiantes que se trasladan $(32,9 \%)$, que los que atienden los municipalizados $(25,5 \%)$ y los particulares pagados $(24,4 \%)$. En cuanto a los GSE, son los establecimientos de los sectores medios los que reciben a una mayor proporción de trasladados; en cambio, son los GSE extremos (bajo y alto) los que atienden proporcionalmente a menos estudiantes que se trasladan.

\section{Tabla 4}

Distribución de los estudiantes que se trasladan en cada dependencia y GSE

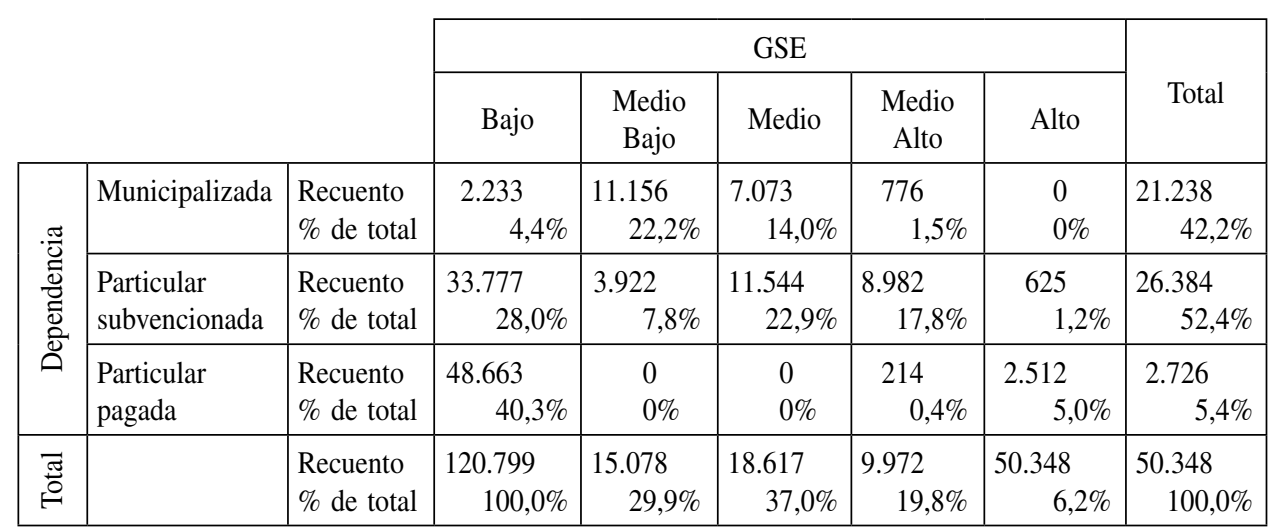


Es interesante observar los establecimientos donde hay menos alumnos trasladados proporcionalmente. En esta categoría coinciden dos grupos de establecimientos que socialmente son muy distantes entre sí: los del GSE bajo del municipalizado $(18,1 \%)$ y el alto del particular pagado $(23,4 \%)$.

Con respecto a los resultados de la prueba SIMCE de los establecimientos (ver tabla 6), se puede constatar que la mayor cantidad de alumnos que se trasladan asisten a establecimientos que logran un desempeño significativamente menor que el de sus similares: esto es, 20.057 estudiantes, que corresponde al 40,8\% de todos los trasladados. Los estudiantes trasladados que asisten a establecimientos de igual desempeño son el $27,2 \%$ y a establecimientos de mayor desempeño, el 32,0\%. Las diferencias en la distribución de los trasladados en los diversos niveles de desempeño SIMCE son estadística y sustantivamente significativas $\left(\chi^{2}=1.468,65\right.$, Sig. $\left.=0,000\right)$. Cabe destacar que esta situación se repite en las tres dependencias y en los cinco GSE.

Tabla 5

Proporción de alumnos trasladados en cada dependencia y GSE

\begin{tabular}{|l|c|c|c|c|c|c|}
\hline Dependencia/GSE & Bajo & Medio bajo & Medio & Medio alto & Alto & Total \\
\hline Municipalizado & $18,1 \%$ & $25,9 \%$ & $27,9 \%$ & $29,6 \%$ & & $25,5 \%$ \\
\hline P. subvencionado & $38,5 \%$ & $36,4 \%$ & $31,5 \%$ & $32,6 \%$ & $33,2 \%$ & $32,9 \%$ \\
\hline P. pagado & & & & $48,9 \%$ & $23,4 \%$ & $24,4 \%$ \\
\hline Total & $22,5 \%$ & $28,0 \%$ & $30,0 \%$ & $32,6 \%$ & $24,9 \%$ & $27,5 \%$ \\
\hline
\end{tabular}

\section{Tabla 6}

Distribución de los estudiantes que permanecen y de los trasladados en los distintos niveles de desempeño SIMCE

\begin{tabular}{|c|c|c|c|c|c|}
\hline & & & \multicolumn{2}{|c|}{ Movilidad Escolar } & \multirow{2}{*}{ Total } \\
\hline & & & Permanecen & Se trasladan & \\
\hline \multirow{3}{*}{$\sum_{\Omega}^{U}$} & Menor & $\begin{array}{l}\text { Recuento porcentaje } \\
\text { de Movilidad Escolar }\end{array}$ & $\begin{array}{r}38.359 \\
31,8 \% \\
\end{array}$ & $\begin{array}{r}20.057 \\
40,8 \% \\
\end{array}$ & $\begin{array}{r}58.416 \\
34,4 \% \\
\end{array}$ \\
\hline & Igual & $\begin{array}{l}\text { Recuento porcentaje } \\
\text { de Movilidad Escolar }\end{array}$ & $\begin{array}{r}33.777 \\
28,0 \% \\
\end{array}$ & $\begin{array}{r}13.404 \\
27,2 \% \\
\end{array}$ & $\begin{array}{l}47.181 \\
27,8 \% \\
\end{array}$ \\
\hline & Mayor & $\begin{array}{l}\text { Recuento porcentaje } \\
\text { de Movilidad Escolar }\end{array}$ & $\begin{array}{l}48.663 \\
\quad 40,3 \%\end{array}$ & $\begin{array}{l}15.736 \\
32,0 \%\end{array}$ & $\begin{array}{r}64.399 \\
37,9 \% \\
\end{array}$ \\
\hline 营 & & $\begin{array}{l}\text { Recuento porcentaje } \\
\text { de Movilidad Escolar }\end{array}$ & $\begin{array}{l}120.799 \\
100,0 \%\end{array}$ & $\begin{array}{l}49.197 \\
100,0 \%\end{array}$ & $\begin{array}{l}169.996 \\
100,0 \%\end{array}$ \\
\hline
\end{tabular}


Por otra parte, es importante considerar que la distribución de la población, sumando a los que permanecen y se trasladan, no es similar en los tres niveles de desempeño (ver tabla 6, columna derecha). Si se controla esta distribución desigual, la proporción de estudiantes trasladados a establecimientos de menor SIMCE es más pronunciada aún.

Para controlar el efecto del desigual tamaño en cada grupo de desempeño SIMCE, a continuación se examina la proporción de estudiantes trasladados en cada nivel de desempeño, considerando dependencia y GSE (ver tabla 7). Este análisis permite identificar el tipo de establecimiento que, proporcionalmente, atiende más estudiantes trasladados. La tabla 7 agrega también la correlación ( $\mathrm{r}$ de Pearson) entre porcentaje de alumnos trasladados del establecimiento y puntaje promedio SIMCE de éste.

Considerando el total de la población (tabla 7, columna derecha, última fila), se puede constatar que en general los establecimientos de menor desempeño tienen una mayor proporción de estudiantes trasladados $(34,3 \%)$, que los de igual o de mejor desempeño $(28,4 \%$ y $24,4 \%$ respectivamente). Es decir, en tanto aumenta el rendimiento SIMCE de un colegio, disminuye la proporción de alumnos trasladados que se reciben. Esta situación se presenta en todas las dependencias y GSE.

\section{Tabla 7}

Proporción de alumnos trasladados en cada nivel de desempeño SIMCE, considerando dependencia y GSE

\begin{tabular}{|c|c|c|c|c|c|c|}
\hline Dependencia/GSE & Bajo & Medio bajo & Medio & Medio alto & Alto & Total \\
\hline $\begin{array}{l}\text { Municipalizado Menor } \\
\text { SIMCE } \\
\text { Igual SIMCE } \\
\text { Mayor SIMCE }\end{array}$ & $\begin{array}{r}22,0 \% \\
16,9 \% \\
17,2 \% \\
\mathrm{r}=-0.183^{* *}\end{array}$ & $\begin{array}{c}30,7 \% \\
24,2 \% \\
22,1 \% \\
\mathrm{r}=-0.277 * *\end{array}$ & $\begin{array}{r}30,1 \% \\
27,6 \% \\
24,6 \% \\
r=-0.216^{* *}\end{array}$ & $\begin{array}{r}32,2 \% \\
36,7 \% \\
24,2 \% \\
r=-0.162 * *\end{array}$ & & $\begin{array}{r}29,8 \% \\
24,3 \% \\
22,2 \% \\
r=-.183^{* *}\end{array}$ \\
\hline $\begin{array}{l}\text { P. Subvencionado Menor } \\
\text { SIMCE } \\
\text { Igual SIMCE } \\
\text { Mayor SIMCE }\end{array}$ & $\begin{aligned} 41,9 \% \\
39,2 \% \\
32,1 \% \\
\mathrm{r}=-0.153^{* *}\end{aligned}$ & $\begin{aligned} 45,0 \% \\
37,2 \% \\
30,4 \% \\
\mathrm{r}=-0.287 * *\end{aligned}$ & $\begin{aligned} & 39,8 \% \\
& 31,6 \% \\
& 26,4 \% \\
& r=-0.351 * *\end{aligned}$ & $\begin{array}{r}38,8 \% \\
35,6 \% \\
25,6 \% \\
\mathrm{r}=-0.315^{* *}\end{array}$ & $\begin{aligned} 45,0 \% \\
21,7 \% \\
29,0 \% \\
\mathrm{r}=-0.550 * *\end{aligned}$ & $\begin{array}{c}40,4 \% \\
33,8 \% \\
26,9 \% \\
r=-.275^{* *}\end{array}$ \\
\hline $\begin{array}{l}\text { P. Pagado Menor SIMCE } \\
\text { Igual SIMCE } \\
\text { Mayor SIMCE }\end{array}$ & & & & $\begin{array}{r}50,0 \% \\
45,3 \% \\
46,6 \% \\
\mathrm{r}=-0.218^{* *}\end{array}$ & $\begin{array}{c}33,9 \% \\
22,8 \% \\
17,0 \% \\
\mathrm{r}=-0.446^{* *}\end{array}$ & $\begin{array}{r}34,8 \% \\
23,2 \% \\
18,3 \% \\
r=-.473 * *\end{array}$ \\
\hline $\begin{array}{l}\text { Total Menor SIMCE } \\
\text { Igual SIMCE } \\
\text { Mayor SIMCE }\end{array}$ & $\begin{array}{c}28,0 \% \\
22,0 \% \\
18,8 \% \\
\mathrm{r}=-0.238^{* *}\end{array}$ & $\begin{array}{c}33,0 \% \\
26,3 \% \\
24,3 \% \\
\mathrm{r}=-0.249 * *\end{array}$ & $\begin{aligned} 34,5 \% \\
30,1 \% \\
25,9 \% \\
r=-0.261 * *\end{aligned}$ & $\begin{array}{r}38,3 \% \\
35,8 \% \\
25,7 \% \\
\mathrm{r}=-0.298 * *\end{array}$ & $\begin{array}{c}36,7 \% \\
22,6 \% \\
17,9 \% \\
\mathrm{r}=-0.482 * *\end{array}$ & $\begin{aligned} & 34,3 \% \\
& 28,4 \% \\
& 24,4 \% \\
& \mathrm{r}=-0.156^{* *}\end{aligned}$ \\
\hline
\end{tabular}

** Se establece la correlación ( $\mathrm{r}$ de Pearson) entre porcentaje de alumnos trasladados del establecimiento y puntaje SIMCE, Lenguaje y Comunicación, que el colegio obtiene. En todos los casos la correlación es significativa al nivel 0,01 . 
Esta relación se aprecia también en la correlación (inversa) entre puntaje SIMCE del establecimiento y el porcentaje de estudiantes trasladados que atienden. La correlación alcanza para el total de la población un valor significativo de $r-0.156$. Ello significa que mientras mayor es el puntaje SIMCE, menor es el porcentaje de estudiantes trasladados que éste recibe.

Es importante destacar que esta relación (inversa) está cruzada por el GSE del establecimiento. Se puede observar (tabla 7, última fila) que mientras mayor es el GSE, mayor es la correlación: en el GSE Bajo el $r$ de Pearson toma un valor de -0,238; y en el GSE Alto alcanza un $\mathrm{r}$ de -0,482. Ello significa que mientras más alto es el GSE del establecimiento, más fuerte es la asociación entre mejor SIMCE y menor porcentaje de trasladados. Al respecto, es interesante destacar el caso del GSE Alto, donde los establecimientos de mejor SIMCE tienen un $17,9 \%$ de alumnos trasladados, en cambio los de menor SIMCE doblan este porcentaje llegando a un 36,7\%.

Lo anterior implica que es más difícil ingresar a un establecimiento de alto SIMCE que ingresar a uno de bajo desempeño; y que esta dificultad es aún mayor cuando aumenta el GSE del establecimiento.

A esta dificultad también se puede agregar el factor dependencia: la correlación más alta se presenta en los particulares pagados $(r=-0,473)$, la cual prácticamente dobla el peso de la correlación de las otras dependencias.

El análisis anterior considera el promedio SIMCE de todos los estudiantes; esto es, de los trasladados y de los que permanecen. Este promedio podría tener la dificultad de confundir la calidad educativa que ofrece el establecimiento de destino con la calidad del establecimiento de origen de los estudiantes trasladados. Cabe señalar que muchos colegios tienden a diferenciar el desempeño de los alumnos que han formado desde el inicio de la escolaridad de aquellos que no se han iniciado con ellos. Para controlar este efecto, a continuación se reagrupan los establecimientos por nivel de desempeño SIMCE considerando sólo los resultados de los alumnos que han permanecido en el mismo establecimiento desde $1^{\circ}$ E.B. o antes. La distribución de los estudiantes traslados se presentan en la siguiente tabla (8)

\section{Tabla 8}

Distribución de los estudiantes que permanecen y de los trasladados en los distintos niveles de desempeño SIMCE -considerando el resultado de los alumnos que permanecen-*

\begin{tabular}{|c|c|c|c|c|c|}
\hline & & & \multirow{2}{*}{\multicolumn{2}{|c|}{ Movilidad Escolar }} & \multirow{3}{*}{ Total } \\
\hline & & & & & \\
\hline & & & Permanecen & Se trasladan & \\
\hline \multirow{3}{*}{$\sum_{\text {U }}^{\text {U⿱山己心 }}$} & Bajo & $\begin{array}{l}\text { Recuento porcentaje } \\
\text { de Movilidad Escolar }\end{array}$ & $\begin{array}{r}33.759 \\
28,2 \%\end{array}$ & $\begin{array}{r}18.110 \\
37,3 \%\end{array}$ & $\begin{array}{r}51.869 \\
30,8 \%\end{array}$ \\
\hline & Mediano & $\begin{array}{l}\text { Recuento porcentaje } \\
\text { de Movilidad Escolar }\end{array}$ & $\begin{array}{l}44.345 \\
37,0 \%\end{array}$ & $\begin{array}{r}17.177 \\
35,4 \%\end{array}$ & $\begin{array}{r}61.522 \\
36,5 \%\end{array}$ \\
\hline & Alto & $\begin{array}{l}\text { Recuento porcentaje } \\
\text { de Movilidad Escolar }\end{array}$ & $\begin{array}{r}41.605 \\
34,8 \%\end{array}$ & $\begin{array}{r}13.273 \\
32,0 \%\end{array}$ & $\begin{array}{r}54.938 \\
32,6 \%\end{array}$ \\
\hline ప్ & & $\begin{array}{l}\text { Recuento porcentaje } \\
\text { de Movilidad Escolar }\end{array}$ & \begin{tabular}{|c|}
119.769 \\
$100,0 \%$
\end{tabular} & $\begin{array}{l}48.560 \\
100,0 \%\end{array}$ & $\begin{array}{r}168.329 \\
100,0 \%\end{array}$ \\
\hline
\end{tabular}

* La clasificación en Bajo, Mediano y Alto se hace de acuerdo a los resultados obtenidos sólo por los estudiantes que han permanecido en el mismo establecimiento desde $1^{\circ}$ E.B. o antes. 
Se puede constatar que los estudiantes que se trasladan lo hacen preponderantemente hacia establecimientos donde sus pares obtienen bajo desempeño SIMCE. El 37,3\% de todos los trasladados (18.110 estudiantes) se ubican en establecimientos de menor SIMCE; el $35,4 \%$ en los de mediano SIMCE y el $27,3 \%$ en los de alto SIMCE. Las diferencias en la distribución de los trasladados en los diversos niveles de desempeño son estadística y sustantivamente significativas $\left(\chi^{2}=1.544,14\right.$ Sig. $\left.=0,000\right)$. Esta situación se presenta en las tres dependencias y en los cinco GSE. La magnitud de las diferencias es más relevante aún si se considera que la distribución de la población en los tres niveles no es igual (ver tabla 8, columna derecha).

$\mathrm{Al}$ igual como se hizo en los análisis anteriores, a continuación se examinan la proporción de alumnos traslados en cada grupo de desempeño SIMCE y la correlación entre porcentaje de alumnos trasladados y puntaje del establecimiento (tabla 9).

En términos generales, se constata que los establecimientos que presentan menor desempeño SIMCE (en sus alumnos originarios) tienen un mayor porcentaje de estudiantes trasladados $(34,9 \%)$ que los de alto desempeño (24,2\% de trasladados). Ello se aprecia también en la correlación (inversa) entre desempeño SIMCE y porcentaje de trasladados que es estadísticamente significativa $(\mathrm{r}=-0.075$; sig. 0.01). Al respecto, es justificable señalar que los estudiantes que se trasladan tienden a movilizarse, en una mayor proporción, hacia establecimientos donde sus pares presentan menor desempeño académico.

Esta situación adquiere mayor fuerza en la medida que aumenta el GSE del establecimiento. Es decir, mientras más alto es el GSE, más fuerte es que los trasladados se ubiquen en los establecimientos con menores desempeños. Esta tendencia se torna

\section{Tabla 9}

Proporción de alumnos trasladados en cada nivel de desempeño SIMCE -de los estudiantes que permanecen- considerando dependencia y GSE

\begin{tabular}{|l|c|c|c|c|c|c|}
\hline Dependencia/GSE & Bajo & Medio bajo & Medio & Medio alto & Alto & Total \\
\hline Municipalizado Bajo SIMCE & $21,4 \%$ & $30,9 \%$ & $30,0 \%$ & $32,5 \%$ & & $29,6 \%$ \\
Mediano SIMCE & $17,6 \%$ & $24,0 \%$ & $28,1 \%$ & $34,9 \%$ & & $24,3 \%$ \\
Alto SIMCE & $15,6 \%$ & $21,5 \%$ & $23,7 \%$ & $22,2 \%$ & & $21,2 \%$ \\
& $\mathrm{r}=-0.073^{* *}$ & $\mathrm{r}=-0.185^{* *}$ & $\mathrm{r}=-0.238^{* *}$ & $\mathrm{r}=-0.297$ & & $\mathrm{r}=-0.109 * *$ \\
\hline P. Subvencionado Bajo SIMCE & $42,5 \%$ & $47,1 \%$ & $42,1 \%$ & $40,3 \%$ & $50,9 \%$ & $42,6 \%$ \\
Mediano SIMCE & $37,5 \%$ & $33,9 \%$ & $31,5 \%$ & $33,7 \%$ & $18,2 \%$ & $32,5 \%$ \\
Alto SIMCE & $31,2 \%$ & $30,9 \%$ & $26,3 \%$ & $26,6 \%$ & $31,0 \%$ & $27,1 \%$ \\
& $\mathrm{r}=-0.135^{* *}$ & $\mathrm{r}=-0.197 * *$ & $\mathrm{r}=-0.340^{* *}$ & $\mathrm{r}=-0.286 * *$ & $\mathrm{r}=-0.520 * *$ & $\mathrm{r}=-0.175^{* *}$ \\
\hline P. Pagado Bajo SIMCE & & & & $51,6 \%$ & $37,5 \%$ & $38,9 \%$ \\
Mediano SIMCE & & & & $61,0 \%$ & $23,1 \%$ & $23,5 \%$ \\
Alto SIMCE & & & & $43,3 \%$ & $17,9 \%$ & $18,9 \%$ \\
& & & & $\mathrm{r}=-0.399 *$ & $\mathrm{r}=-0.402 * *$ & $\mathrm{r}=-0.447 * *$ \\
\hline Total Bajo SIMCE & & & & $39,7 \%$ & $41,1 \%$ & $34,9 \%$ \\
Mediano SIMCE & $28,0 \%$ & $33,6 \%$ & $35,1 \%$ & $39 \%$ & $22,3 \%$ & $27,9 \%$ \\
Alto SIMCE & $21,4 \%$ & $25,8 \%$ & $30,1 \%$ & $33,9 \%$ & $18,7 \%$ & $24,2 \%$ \\
& $17,9 \%$ & $23,8 \%$ & $25,6 \%$ & $26,5 \%$ & $\mathrm{r} \%$ \\
\hline
\end{tabular}

* La correlación es significativa al nivel 0,05 (bilateral).

** La correlación es significativa al nivel 0,01 (bilateral). 
especialmente significativa en los establecimientos del GSE alto, donde el porcentaje de alumnos trasladados en los establecimientos de bajo SIMCE está cerca de doblar a los de alto desempeño. Ello se aprecia también en los valores que toma la correlación: en el GSE alto del particular subvencionado alcanza un $\mathrm{r}$ de -0.520 (sig. 0.01) y en el particular pagado un $\mathrm{r}$ de -0.447 (sig. 0.01).

Es importante destacar que en los establecimientos municipalizados se presenta la menor correlación entre porcentaje de trasladados y desempeño SIMCE $(r=-0.109)$. Estos datos dan cuenta que los municipalizados logran, en alguna medida, atenuar la tendencia de que los trasladados se ubiquen principalmente en los establecimientos de peores resultados.

Finalmente, cabe indicar que existe una consistencia entre los datos referidos a los niveles de desempeño considerando sólo a los alumnos que permanecen y los presentados en el análisis anterior (SIMCE de todos los estudiantes; ver tabla 7).

\section{CONCLUSIONES}

La movilidad escolar es un fenómeno altamente recurrente en Chile. Los niños y niñas de $4^{\circ}$ E.B. que han tenido la experiencia de trasladarse de un establecimiento a otro suman 50.348 estudiantes. Esta cifra representa el $27,5 \%$ de la población escolar de este nivel.

La perspectiva neoliberal de la educación, que ha operado en Chile desde hace 30 años, valora positivamente la movilidad debido a que los "clientes" de la educación están haciendo uso de su libertad y racionalidad para trasladarse a una mejor alternativa. Asimismo, se considera que la alta movilidad potencia el desarrollo de un mercado más eficiente, donde las mejores escuelas logran atraer a una mayor demanda y las peores tienden a desaparecer debido a que no son elegidas.

Sin embargo, ¿se pueden sostener estos planteamientos a la luz de los resultados obtenidos en este estudio? Desde esta evidencia, ¿qué implicancias tiene la movilidad escolar en la calidad y equidad de la educación chilena?

A continuación se desarrollan tres conclusiones relativas a estas interrogantes. La primera se refiere a las implicancias de la movilidad en los aprendizajes de los estudiantes. La segunda alude a las implicancias de la movilidad en la calidad que ofrecen los establecimientos. La tercera analiza las implicancias para la integración y equidad social.

\section{A) IMPLICANCIAS DE LA MOVILIDAD EN EL APRENDIZAJE}

Desde una lógica de mercado la movilidad escolar es positiva en cuanto permite a los estudiantes transitar de un establecimiento de peor calidad a otro mejor y, por consiguiente, mejorar sus aprendizajes.

De acuerdo a los datos recogidos hay importantes diferencias en los desempeños académicos de los alumnos que se trasladan y los que permanecen. Los estudiantes que se trasladan obtienen 5,74 puntos menos en la prueba SIMCE que los estudiantes que permanecen. En todos los grupos socioeconómicos y en todas las dependencias se presentan diferencias significativas, situándose los que se trasladan en una peor posición. 
Esta información hay que analizarla con detención. Una primera lectura puede llevar a plantear que el traslado es en sí mismo una dificultad para el aprendizaje pues los alumnos bajan su desempeño. Sin embargo, los datos no son suficientes para llegar a tal conclusión. El presente estudio no examina el desempeño histórico de los alumnos; no se sabe si los peores resultados se deben al cambio o son un problema anterior al traslado. Sólo, hasta ahora, podemos constatar que los estudiantes que se trasladan presentan, en promedio, más dificultades en sus aprendizajes que los que permanecen.

Una problemática que sí se puede desprender desde la evidencia obtenida se refiere al tipo de establecimiento en donde se ubican los estudiantes que se trasladan. Los resultados indican que se concentran principalmente $(40,8 \%$ de los estudiantes que se trasladan) en los establecimientos que logran menor SIMCE, controlado el grupo socioeconómico. Así también, la mayor proporción de los traslados (37,3\%) asisten a establecimientos en donde el desempeño de sus compañeros, los que han permanecido desde $1^{\circ}$ E.B. en la misma escuela, es significativamente menor al que obtienen los niños del mismo grupo socioeconómico.

En suma, se puede afirmar que los estudiantes que se trasladan tienden a presentar peores desempeños académicos que los que permanecen y tienden a ubicarse en establecimientos de menor calidad comparado con otros del mismo grupo socioeconómico, y con compañeros de curso que presentan también bajos resultados académicos.

Por consiguiente, se puede concluir que la movilidad escolar que opera actualmente en Chile, puede significar una dificultad para el aprendizaje de los alumnos en cuanto ella implica una trayectoria hacia contextos educativos menos favorables. Lo anterior muestra la debilidad del planteamiento que indica que la movilidad es una opción familiar, que busca de modo racional y libre algo mejor. Por lo visto, la movilidad representa para las familias una peor alternativa, donde sus hijos con dificultades tienden a optar por los peores establecimientos, pudiéndolos debilitar aún más en sus aprendizajes.

\section{B) IMPLICANCIAS DE LA MOVILIDAD EN LA CALIDAD DE LOS ESTABLECIMIENTOS}

Desde la lógica de mercado, la movilidad escolar es positiva porque presiona para que los establecimientos de peor calidad desaparezcan por falta de interesados y se robustezcan los mejores.

Como ya se indicó, la evidencia muestra que el mayor porcentaje de los estudiantes que se trasladan $(40,8 \%)$ asisten a establecimientos que logran un desempeño significativamente peor que el de sus similares. También es relevante destacar que la proporción de estudiantes trasladados en las escuelas de peor SIMCE es significativamente más alta $(34,3 \%)$ que la que tienen los mejores colegios $(24,2 \%)$.

Lo anterior indica que los colegios de peor desempeño académico son los que más atraen a los estudiantes que se trasladan. Y al contrario, en la medida que los colegios mejoran sus resultados, disminuye la cantidad de estudiantes trasladados que reciben. La correlación alcanza a un 0.156 , siendo estadísticamente significativa.

Esta situación da cuenta que no es real que los establecimientos que presentan peores resultados tiendan a desaparecer sólo por efecto de falta de alumnos matriculados. La alta movilidad escolar que se presenta en Chile los ha beneficiado, constituyéndose estos colegios en la alternativa más frecuente para los que se trasladan. 
Esta situación podría ser explicada por múltiples factores: falta de información de los padres acerca del colegio que trasladan a sus hijos, escasez de cupos de los mejores establecimientos, requisitos y trabas que ponen los mejores para seleccionar a los nuevos alumnos, autoselección de las familias, etc. En futuras investigaciones habrá que examinar estos y otros factores. No obstante, con la información disponible, se puede concluir que la alta movilidad escolar no se asocia a la mejora de la calidad académica de los establecimientos: las familias, por diversas razones, tienden a optar por los peores colegios. La confianza de que estos colegios desaparecerán por falta de interesados, es más un anhelo que una realidad.

\section{C) IMPLICANCIAS DE LA MOVILIDAD EN LA INTEGRACION SOCIAL}

La evidencia recogida indica que la movilidad escolar opera en forma distinta en los diversos grupos socioeconómicos. La mayor movilidad se presenta en los sectores medios y la menor en los grupos extremos. Asimismo, los grupos socioeconómicos alto y bajo son los que reciben a una menor proporción de estudiantes trasladados. Destacan al respecto los municipalizados del GSE Bajo (18,1\% de alumnos trasladados) y el particular pagado del GSE alto (23,4\% de trasladados). Estos datos permiten concluir que en Chile se presenta una tendencia a un cierre social en los establecimientos de los sectores extremos. Es más difícil trasladarse a ellos, que hacerlos en los sectores medios.

Asimismo, es interesante advertir que este cierre no es el mismo para los colegios más pobres que para los más acomodados. La evidencia indica que en los establecimientos más acomodados existe una alta relación entre resultado SIMCE y porcentaje de alumnos trasladados $(\mathrm{r}-0,482)$. Esto quiere decir que mientras aumentan los resultados académicos de los establecimientos, al mismo tiempo disminuye la cantidad de alumnos trasladados que reciben; o a la inversa, mientras peores son los resultados, más alumnos traslados reciben. Los establecimientos más pobres también presentan una correlación significativa, pero ella es sustantivamente más débil que la anterior. Estos datos indican que los colegios de los grupos acomodados no sólo están más cerrados a la movilidad escolar, característica que comparten con los más pobres, sino además tienden a diferenciarse fuertemente entre ellos, siendo los establecimientos de menor calidad los que se encargan de recibir a los trasladados. En cambio, en los establecimientos más pobres, la distribución de los trasladados en más equitativa. El porcentaje de trasladados de los establecimientos de menor SIMCE $(22,0 \%)$ es bastante cercano al porcentaje que reciben los de mayor SIMCE $(17,2 \%)$.

A partir de lo anterior se puede concluir que la movilidad escolar que opera en Chile no constituye un aporte significativo a la integración social. Los grupos extremos tienden a cerrarse y en los sectores más acomodados se agrega una nueva diferenciación: la ubicación de los trasladados en este selecto grupo socioeconómico se hace principalmente en los que ofrecen peor calidad. Por consiguiente, la movilidad escolar, tal como opera en Chile, tiende a profundizar las distancias sociales y las diferencias académicas. Los mejores colegios, donde se educan los sectores privilegiados, no son una real alternativa en el mercado escolar: lo que significa que "los mejores" colaboran para que la elección de las familias sea principalmente por los "peores". 


\section{REFERENCIAS BIBLIOGRAFICAS}

Aedo, C. \& Sapelli, C. (2001). El sistema de vouchers en educación: una revisión de la teoría y evidencia empírica para Chile. Estudios Públicos, 82, 35-82.

Consejo Asesor Presidencial para la Calidad de la Educación (2006). Informe Final. Santiago, Chile: Gobierno de Chile.

Eyzaguirre, B. \& Fontaine, L. (2008). Las escuelas que tenemos. Santiago, Chile: CEP.

Fontaine, L. \& Eyzaguirre, B. (2001). Una estructura que presione a las escuelas a hacerlo bien. Santiago, Chile: CEP.

Friedman, M. y Friedman, R. (1980). Libertad de elegir. Barcelona: Grijalbo.

Hanushek, E., Kain, J. \& Rivkin, S. (2004). Disruption versus Tiebout improvement: the costs and benefits of switching schools. Journal of Public Economics 88 (2004) 1721-1746.

JUNAEB (2003). Comprendiendo el fenómeno de la deserción escolar en Chile. Santiago de Chile: JUNAEB.

Manzi, J. (2007). Selección de estudiantes en el sistema escolar chileno: ¿la buena educación debe ser sólo para los mejores? En: Bruner y Peña (ed.). La reforma al sistema escolar. Aportes para el debate. Santiago, Chile: Universidad Diego Portales, Universidad Adolfo Ibáñez.

March, J. y Simon, H. (1977). Teoría de la organización. Barcelona: Ariel.

March, J. (1994). A primer on decision making: How decisions happen. New York: The Free Press.

Mena, M.I., Milicic, N., Romagnoli, C. y Valdés, A.M. (2006). Antecedentes que avalan la importancia y urgencia de integrar al currículum escolar la formación socioafectiva, ética y de la convivencia social. Santiago: Documento Valoras UC.

Milicic, N. y Aron, A. (1996). Desarrollo social y desarrollo cognitivo: un programa de mejoramiento del clima social escolar. En Beas, J. (ed.). Enfoques cognitivos actuales en educación. Santiago: Facultad de Educación de la Pontificia Universidad Católica de Chile.

Ministerio de Educación de Chile (2007). Base de Datos SIMCE $4^{o}$ año de Enseñanza Básica. Santiago de Chile: MINEDUC.

OCDE (2004). Informe del sistema educativo chileno. Santiago de Chile.

Peña, C. (2007). Por qué no debemos seleccionar. En: Bruner, J.J. y Peña, C. (ed.). La reforma al sistema escolar. Aportes para el debate. Santiago, Chile: Universidad Diego Portales, Universidad Adolfo Ibáñez.

Perticara, M.W. y Sanclemente, M. (2009). ¿Pueden sus padres elegir un buen colegio? Revista Observatorio Económico, $\mathrm{N}^{\circ} 28$.

Raczynski, D. et al. (2002). Procesos de Deserción en la Educación Media. Factores Expulsores y Protectores. Santiago, Chile: INJUV.

República de Chile. Ley General de Educación (2009).

Sanclemente, M. (2008). Nomadismo escolar en el sistema educacional chileno (2003-2007). Monografía de Graduación realizada como parte de los requerimientos para obtener el grado de Master of Arts in Economics, Georgetown University; Universidad Alberto Hurtado. 
